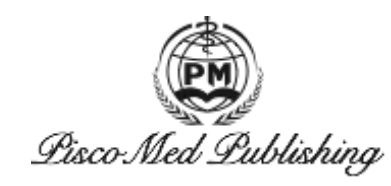

Original Research Article

\title{
Present Situation and Countermeasure of Camellia Oleifera Industry in Guizhou Province
}

Yang Long*

School of Economics and Management, Tongren University, Guizhou 554300, China. E-mail: 326490228@qq.com

Abstract: Starting from the current situation of the development of camellia oleifera industry in Guizhou Province, China, this paper systematically summarizes the development advantages, and puts forward the countermeasures for further development. Moreover, it points out that camellia oleifera industry must strengthen the propaganda, put the promotion of science and technology in the first place, deal with a series of problems such as variety resources, planting technology, capital investment, low-yield forest transformation, and processing of camellia oleifera, so as to speed up the processing development of camellia oleifera industry in Guizhou Province.

Keywords: Camellia Oleifera Industry; Current Situation; Advantages; Countermeasures

Guizhou Province is located in the southwest of China. Farmers in the mountainous areas have always had the traditional habits of planting and eating camellia oleifera. The local climate and geographical conditions are very suitable for the growth of camellia oleifera. At present, the production of camellia oleifera has become an important content of "Mountain Reconstruction", which is also an important source of income for farmers in Guizhou mountainous areas. So it has become an important project that is valued and supported by governments at all levels. However, due to the lack of investment in capital and technology in the production of camellia oleifera in Guizhou Province in the past, at present tending camellia oleifera forest is usually carried out by farmers spontaneously.

\section{Current situation of camellia oleifera industry in Guizhou Province}

Guizhou Province is one of the important camellia oleifera producing areas in Southern China. It is of great significance to make full use of the ecological, economic and social benefits of camellia oleifera, accelerate the development of camellia oleifera industry, promote the economic development of mountainous areas in Guizhou Province, promote the income of farmers, maintain the security of national grain and oil, so as to meet the needs of society and the public for good ecological products and natural green products.

\subsection{Guizhou Province with a long history of planting camellia oleifera}

There is a long history of planting camellia cultivation in Guizhou Province, many counties and cities of which are well-known as the "Hometown of Camellia Oleifera", such as Wangmo, Ceheng, Tianzhu and Yuping. At present, camellia oleifera forest that are over 100 years can be seen everywhere. These camellia oleifera forests are ancestral for the whole family to eat all year round, who inherit a whole set of skills of selecting seed, harvesting and processing. Camellia oleifera in Ceheng, Luojiang, Liping Ouyang and Shuikou is in good condition, which is an important economic source of family income.

\footnotetext{
Copyright (C) 2020 Yang Long

doi: $10.18282 /$ ff.v9i1.831

This is an open-access article distributed under the terms of the Creative Commons Attribution Non-Commercial License

(http://creativecommons.org/licenses/by-nc/4.0/), which permits non-commercial use, distribution, and reproduction in any medium, provided the original work is properly cited.
} 


\subsection{Strict supervision on quality of camellia oleifera seedlings}

In order to strengthen the quality management of camellia oleifera seedlings, ensure the supply and the healthy development of camellia oleifera industry in Guizhou Province, according to Regulation on Quality Management of Camellia Oleifera Seedlings issued by the State Forestry Administration, combined with the key development plan of camellia oleifera industry in Guizhou Province, on the basis of local application, the first batch of camellia oleifera seedlings in Guizhou Province has been determined through full investigation and research with strict screening and scientific evaluation. There are twelve designated production units and one cutting garden for improved varieties of camellia oleifera. Seven places in Qiandongnan Prefecture are listed as the first batch of the fixed-point production unit of camellia seeding nursery and ear picking nursery. There were more than 7.1 million grafted seedlings only in Tianzhu County in 2009, while counties like Liping, Songtao, Ceheng, and Wangmo have successively built ear picking nurseries.

\subsection{Getting attention from governments at all levels}

Camellia oleifera is a major characteristic and advantageous resource in the forestry construction of Guizhou Province. But for a long time, there has been the following problems, namely aging, extensive production and management, mixed varieties, low productivity and economic benefits. It is necessary to make full use of the long history and good production foundation of camellia oleifera in Tongren, Qiandongnan and Qianxinan. In 2009, the Provincial Forestry Department issued The Implementation Opinions of Guizhou Provincial Forestry Department on Accelerating the Development of Camellia Oleifera Industry, so as to make full use of the characteristic resource advantages of camellia oleifera and promote the rapid development of camellia oleifera industry in Guizhou Province.

\subsection{Organizing technology training}

The provincial quality management and technology training meeting of camellia oleifera seedlings was held in Tianzhu County. The director of seedling station of Provincial Forestry Department, as well as the relevant leaders of Qiandonnan Forestry Bureau and Tianzhu County attended the meeting for guidance. There were 99 persons that were in charge of forestry seedling and science and technology promotion management departments in nineteen counties (cities) as well as five regions (prefectures and cities) including Qiandongnan, Tongren, Qianxinan, Xingyi and Zunyi. Through technology training, the participants basically mastered the grafting technology of camellia oleifera, which achieved the expected effect of the training meeting and they became the backbone force to promote the development of local camellia oleifera economic industry. By increasing the adjustment of the industrial structure of the camellia oleifera industry, promoting the development of the camellia oleifera industry, it can achieve the forestry efficiency and increase the income of the forest farmers, which can play an exemplary role in the construction of the whole province's camellia oleifera industry.

\subsection{Group company participates in the development of camellia oleifera industry}

Dingzhong Group and Yahong Technology Co., Ltd. that registered in Guizhou Province have invested a lot of money and actively cooperated with local governments to participate and develop camellia oleifera industry. In Tianzhu, Yuping, Ceheng and other main production areas of camellia oleifera, the first step of industrialization of camellia oleifera has been taken. In addition, camellia oleifera processing enterprises such as Dalong and Malinghe in Guizhou Province are also upgrading the processing technology, so as to expand the domestic market and build the enterprise's image.

\section{Countermeasures for the development of camellia oleifera industry in Guizhou Province}

The yield of camellia oleifera per unit area in Guizhou Province is low, so far it is still the rudiment of family self-sufficient individual economy. With the continuous improvement of people's living standards, camellia oleifera has 
gradually penetrated into families. Camellia oleifera has high edible and medicinal value, which has been recognized and accepted by more and more people. Therefore, it can be predicted that the development prospects of domestic and foreign consumer market are quite bright, with the price of camellia oleifera gradually increasing with the passage of time. From the overall situation of the survey, according to the traditional habit of planting camellia oleifera in Guizhou Province, the existing favorable geographical, soil and climatic conditions have been fully used as the basis for the cultivation of camellia oleifera forest, which provide great conditions for the rapid development of camellia oleifera industry in a short period of time. Its potential huge business opportunities can provide a broad sales space for the forest farmers.

\subsection{Planning the layout of camellia oleifera development, promoting the direction of industrialization}

Camellia oleifera has strong adaptability, which has no strict requirements on soil, even tolerates barren. It is suitable to grow in acid yellow soil or red soil with $\mathrm{pH}$ value of 5-6. It requires an annual average temperature of 14-21 ${ }^{\circ} \mathrm{C}$, warm and humid climate, an average temperature of more than $0{ }^{\circ} \mathrm{C}$ in January, and an annual rainfall of over $1000 \mathrm{~mm}$. Guizhou is suitable for the growth of fir, bamboo, pine, camellia oleifera, tea and other trees. It is a land suitable for cultivation and rich in forests. The local soil is mainly thick and medium red soil, red soil and yellow soil. In 2008, the State Forestry Administration put forward the idea of vigorously developing the production of camellia oleifera. The climate, soil and rainfall in Guizhou Province are all favorable for the growth of camellia oleifera forest. In order to effectively develop and tap the potential of forestry production, as well as to achieve the purpose of making the best use of the land and making the best use of the material, the Guizhou Provincial Committee and the government have established the leading position of the production of camellia oleifera, which fully seized the opportunity, and reasonably formulated the general production of camellia oleifera in Guizhou Province, so as to develop the camellia industry as soon as possible and improve its industrialization level.

\subsection{Increasing propaganda of camellia oleifera industry, raising awareness of camellia oleifera industry}

In recent years, the comprehensive utilization of camellia oleifera has a long industrial chain, with great potential for industrial development and broad prospects, but its economic and ecological benefits have not been fully recognized and exploited. The present situation of camellia oleifera production in Guizhou Province can be described as "unknown by others". Therefore, to accelerate the rapid development of the camellia oleifera industry, it is of great practical significance to drive the local farmers to prosper, maintain the security of national grain and oil, promote the construction of new socialist countryside, which can fully meet the needs of the society and the public for natural green products.

By 2020, it will basically realize the intensification of operation and management of the whole province's camellia oleifera industry, the base of resource cultivation, the integration of product development and sales, promote the healthy and sustainable development of the camellia oleifera industry, build three to five leading processing enterprises for refining camellia oleifera processing and series product development, and the annual processing capacity of the whole province's camellia oleifera related enterprises will reach more than 150000 tons. It is estimated that $200000 \mathrm{hm}^{2}$ of newly-built camellia oleifera forest and $66700 \mathrm{hm}^{2}$ of low-yield forest will be completed in the province. The area of camellia oleifera in the whole province has increased from $66700 \mathrm{hm}^{2}$ to $266700 \mathrm{hm}^{2}$. Through the construction of camellia oleifera base and the transformation of low yield forest, the annual average output of camellia oleifera in the whole province has reached 120000 tons and the annual total output value has reached over 10 billion yuan, and the average output of camellia oleifera can reach over $450 \mathrm{~kg} / \mathrm{hm}^{2}$. Therefore, it is necessary to strengthen the publicity of the development of camellia oleifera production, call on all countries (towns) whose conditions permit to establish camellia oleifera demonstration bases, which should widely publicize the importance of the development of camellia oleifera industry to farmers in mountainous areas, and through demonstration publicity, let farmers realize the 
significant benefits of the development of camellia oleifera, so that farmers can actively participate in the development of camellia oleifera industry, and promote the sound and rapid development of camellia oleifera industry.

\subsection{Focusing on supporting leading enterprises, achieving professional development}

The production of camellia oleifera should build the production base of camellia oleifera and solve the supplying problem of the raw material. In order to realize its large-scale development and intensive management, cooperative organizations should be set up to actively support one or two leading enterprises, which can give full play to the guiding and radiating role of cooperative organizations and processing leading enterprises, so as to promote the development of camellia oleifera industry. It is necessary to guide enterprises to participate in the construction of camellia oleifera raw material forest base, promote the industrialized operation mode of "Enterprise + Base + Farmer". Thus it can promote the organic combination of camellia oleifera production, processing as well as market. In order to lead the development of the whole county's camellia oleifera processing industry in terms of radiation and market development, it is necessary to seriously implement the policies and measures to support the development of leading enterprises, making full use of the forestry loan funds in accordance with the principle of "Supporting the large, the strong and the excellent", focusing on supporting capable and skilled people to establish camellia oleifera processing enterprises, increase policy support, and make them continuously expand production scale and develop a professional camellia oleifera processing leading enterprise. To ensure the supply of camellia oleifera raw materials for leading enterprises, small oil extraction workshops should be shut down in a planned way. It is necessary to guide and encourage the cooperation among the camellia oleifera processing enterprises, and work out the relevant policies to improve the market competitiveness together.

\subsection{Vigorously developing base of camellia oleifera forest, accelerating transformation of low yield camellia oleifera forest}

The average investment of planting camellia oleifera forest is about $4500 \mathrm{Yuan} / \mathrm{hm}^{2}$, which is slightly higher than that of commercial timber forest. However, after the planting camellia oleifera, it can blossom and bear fruit in three years or so, and enter the full fruit period in six to seven years, with a life span of over 100 years. Its growth cycle is short and its effect is fast. Moreover, camellia oleifera has a unique advantage due to its low requirements for cultivation technology. The governments at all levels and the competent forestry departments should take the management measures of the combination of the renewal and transformation, the transformation of low-yield forest and the new forest to carry out the production of camellia oleifera, so as to adapt to the current situation and production level of camellia oleifera resources. On the premise of not causing soil erosion, the diseased, old and inferior plants should be rehabilitated and renewed; the existing camellia oleifera forest should be strengthened with young forest tending and mature forest reclamation; the sparse forest land should be replanted; the forest land should be artificially fertilized to prevent diseases and insect pests, so as to improve the planting area and yield of camellia oleifera. To provide farmers with good varieties with high yield, strong resistance to adversity and high oil content, guiding local farmers who are suitable for planting camellia oleifera area to improve the plant density, so as to vigorously plant camellia oleifera, and realize the plant density in $1800-2400$ plants $/ \mathrm{hm}^{2}$, so as to improve the output per unit area and strive to meet the needs of production and development.

The new camellia oleifera forest should make full use of the advantages of capital and technology, so as to accelerate the foundation of camellia oleifera production base. In order to attract more social forces to participate in the construction of camellia oleifera forest, the forestry policy of "Who Plants, Who Owns" should be adhered to. According to the principle of "Law, Compensation and Voluntary", it's necessary to make full use of the achievements of forest right system reform, promote the development of camellia oleifera forest to a large scale, encourage and support all kinds of producers and operators who know technology, have economic strength and are good at management to participate in this industry. Governments at all levels and competent forestry departments should also actively build a high-yield base of camellia oleifera forest, strive for funds from superior departments for agricultural 
comprehensive development projects, or build a demonstration model forest of camellia oleifera, plan and arrange the construction of high-yield forest, combine the projects of returning farmland to forest, poverty alleviation and comprehensive development in mountainous areas, which can play the role of demonstration model, and stimulate the enthusiasm and initiative of the majority of forest farmers to develop camellia oleifera, thus promoting the production of camellia oleifera industry.

\subsection{Building the brand of camellia oleifera of Guizhou Province, promoting the developing strategy of camellia industry}

One of the indispensable components to drive the development of an industry is to rely on brand benefits to improve market competitiveness and create famous brands with local characteristics. Guizhou Province must implement the strategy of famous brand development in order to realize the industrialization of camellia oleifera production. To build a famous brand, it must have a period of accumulation. Therefore, it is necessary to prevent the low standard and low level of repeated construction, keep abreast of the standardized construction in the construction of camellia oleifera base, marketing and product development, thus laying the foundation for the development strategy of camellia oleifera brand in the whole province. The competent forestry departments at all levels in the camellia oleifera producing areas should further recognize the important position and role of the camellia oleifera industry in the development of characteristic commercial forest in Guizhou Province. The leading enterprises should improve their product quality, enhance their brand awareness, give full play to their respective advantages in technology, capital and market, and develop their own famous brand characteristic products, so as to firmly grasp the law of market economic development, and conform to the demand of products from market, and strive to make the camellia oleifera industry in Guizhou Province bigger and stronger.

\section{References}

1. Tan F, Wu D, Huang H. Camellia oleifera in Guizhou: Production situation and development strategies. Journal of Central South Forestry University of Science and Technology 2011; 31(10): 74-77. doi: 10.3969/j.issn.1673-923X.2011.10.013.

2. Luo Z, Qiu J, Xu J, et al. Present situation and countermeasures of the development of camellia oleifera industry in Guizhou Province (in Chinese). Modern Agricultural Technology 2011; (12): 372-374. doi: CNKI:SUN:ANHE.0.2011-12-249.

3. Qiu J, Luo Y, Xu J, et al. Strategy and technical approach to realize the oil camellia industrialization in Guizhou Province. Guizhou Forestry Science and Technology 2013; (4): 51-59. doi: CNKI:SUN:GZLY.0.2013-04-012. 